STRUCTURAL BIOLOGY COMMUNICATIONS

ISSN 2053-230X

\section{Structure of the dual-function fructose-1,6/ sedoheptulose-1,7-bisphosphatase from Thermosynechococcus elongatus bound with sedoheptulose-7-phosphate}

\author{
Charles A. R. Cotton, Burak V. Kabasakal, Nishat A. Miah and James W. Murray*
}

Department of Life Sciences, Imperial College London, Exhibition Road, London SW7 2AZ, England. *Correspondence e-mail: j.w.murray@imperial.ac.uk

The dual-function fructose-1,6/sedoheptulose-1,7-bisphosphatase (FBP/SBPase) in cyanobacteria carries out two activities in the Calvin cycle. Structures of this enzyme from the cyanobacterium Synechocystis sp. PCC 6803 exist, but only with adenosine monophosphate (AMP) or fructose-1,6-bisphosphate and AMP bound. The mechanisms which control both selectivity between the two sugars and the structural mechanisms for redox control are still unresolved. Here, the structure of the dual-function FBP/SBPase from the thermophilic cyanobacterium Thermosynechococcus elongatus is presented with sedoheptulose-7phosphate bound and in the absence of AMP. The structure is globally very similar to the Synechocystis sp. PCC 6803 enzyme, but highlights features of selectivity

at the active site and loop ordering at the AMP-binding site. Understanding the selectivity and control of this enzyme is critical for understanding the Calvin cycle in cyanobacteria and for possible biotechnological application in plants.

\section{Introduction}

The dual-function fructose-1,6/sedoheptulose-1,7-bisphosphatase (FBP/SBPase) plays a role in both the Calvin cycle and gluconeogenesis in cyanobacteria (Tamoi et al., 1996, 1998, 1999). Disruption of the gene encoding the FBP/SBPase is lethal in both Synechococcus sp. PCC 7942 and Synechocystis sp. PCC 6803 (Tamoi et al., 1999). FBP/SBPase is unique in that it catalyses two separate reactions in the Calvin cycle, both of which are catalysed by separate enzymes in plants. The reactions catalysed by $\mathrm{FBP} / \mathrm{SBP}$ ase are important for Calvin cycle flux, as indicated by their high predicted metabolic control coefficients (Poolman et al., 2001). FBP/SBPase has been expressed in transplastomic tobacco (Nicotiana tabacum) and is shown to improve growth under certain circumstances (Miyagawa et al., 2001). The dual-function FBP/SBPase is not regulated in the same way as the corresponding chloroplast enzymes (Tamoi et al., 1998, 1999; Feng et al., 2014). For this reason, the FBP/SBPase has also been the target of algicide screens (Sun et al., 2014) aimed specifically at cyanobacteria. Thermosynechococcus elongatus is a thermophilic cyanobacterium which is used as a model organism for structural analysis of photosynthesis (Murray et al., 2007).

The FBP/SBPase found in $T$. elongatus is a type II FBPase; a member of the larger $\mathrm{Li}^{+}$-sensitive phosphatase superfamily. It shares $80 \%$ (277/347) sequence identity with the Synechocystis sp. PCC 6803 FBP/SBPase. T. elongatus, like 
Table 1

Macromolecule-production information.

Source organism
DNA source
Forward primer
Reverse primer
Expression vector
Expression host
Complete amino-acid sequence
$\quad$ of the construct produced

T. elongatus
T. elongatus genomic DNA
TTCCGCGTGGATCCGTGATAACGTCATC
CTGCAGATCTCGAGTTAGTACAGCTGG
pRSET A
E. coli $($ KRX)
MRGSHHHHHHGLVPRGSMDNVIGLEIIEVVEQAA-
IASARWMGKGDKNMADQAVDAMRNRMNQIHM-
RGRIVIGEGERDEAPMLYIGEEVGICTRPDAA-
QYCNPEELIEIDIAVDPCEGTNLCAYGQPGSM-
AVLAISEKGGLFAAPDFYMKLAAPPAAKGKV-
DIRNSATENLKILSECLDRAIDELVVVVMKRD-
RHNDLIQEIRDAGARVQLISDGDVSAALACAF-
SGTNIHALMGIGAAPEGVISAAAMRALGGHFQ-
GQLVYDPAVVMTKEWANRTREGNLEELKKAGI-
TDPDKVYEAEELASGETVLFAACGITPGMLMK-
GVRFFKGGARTQSLVISTQSKTARFVDTIHMF-
DQQLKSLQLY

Table 2

Crystallization.

\begin{tabular}{|c|c|}
\hline Method & Hanging-drop vapour diffusion \\
\hline Plate type & 24-well plate \\
\hline Temperature (K) & 290 \\
\hline Protein concentration $\left(\mathrm{mg} \mathrm{ml}^{-1}\right)$ & 15 \\
\hline $\begin{array}{l}\text { Buffer composition of protein } \\
\text { solution }\end{array}$ & $\begin{array}{l}50 \mathrm{~m} M \text { Tris- } \mathrm{HCl} \mathrm{pH} \text { 7.9, } 50 \mathrm{~m} M \\
\mathrm{NaCl}, 1 \mathrm{~m} M \text { MgS7P, } 0.2 \mathrm{MgCl}_{2}\end{array}$ \\
\hline Composition of reservoir solution & $\begin{array}{l}0.06 M \text { Na HEPES pH } 7.5,0.12 M \\
\mathrm{MgCl}_{2}, 27 \%(v / v) \text { PEG } 400\end{array}$ \\
\hline Volume and ratio of drop & $2 \mu \mathrm{l}(1: 1)$ \\
\hline Volume of reservoir $(\mu \mathrm{l})$ & 100 \\
\hline
\end{tabular}

Synechocystis sp. PCC 6803, is predicted to have both a type I (tll0541) and type II (tll1276) FBPase. After the Synechocystis sp. PCC $6803 \mathrm{FBP} / \mathrm{SBP} a \mathrm{se}$, the most closely related protein for which there is a structure in the PDB is the class II FBPase GlpX from Escherichia coli ( $42 \%$ sequence identity). The cyanobacterial dual-function FBPases are not regulated by the same mechanisms as cytosolic and eukaryotic FBPases (regulated by AMP, insensitive to DTT) or chloroplastic FBPases (insensitive to AMP, activated by DTT), as they appear to be both activated by DTT and repressed by AMP (Feng et al., 2014).

Both GlpX and the Synechocystis sp. PCC 6803 FBP/ SBPase are compact globular proteins with two $\alpha / \beta$-sandwich domains, but while GlpX is suggested to be active as a dimer (Brown et al., 2009), the active state of the FBP/SBPase is thought to be tetrameric (Feng et al., 2014). The active sites contain three metals; in the case of FBP/SBPase, these are three magnesium ions. The Synechocystis sp. PCC 6803 FBP/SBPase has both an allosteric AMP-binding site and a suggested disulfide bridge (Cys75-Cys99) which might account for dithiothreitol (DTT) sensitivity (Feng et al., 2014), but this disulfide was not observed in the structure and is at an unfeasible distance, with the two thiol groups over $28 \AA$ from one another in crystallographic models (PDB entries 3rpl and 3roj; Feng et al., 2014). The Synechocystis sp. PCC 6803 FBP/ SBPase protein is a biologically active tetramer and residues from two adjacent monomers contribute to the AMP-binding regulatory domain (Arg164, Asn213, His215, Arg307, Phe309
Table 3

Data collection and processing.

Values in parentheses are for the outer shell.

\begin{tabular}{ll}
\hline Diffraction source & Beamline I04, Diamond Light Source \\
Wavelength $(\AA)$ & 0.91730 \\
Temperature $(\mathrm{K})$ & 100 \\
Detector & Pilatus \\
Crystal-to-detector distance (mm) & 326 \\
Rotation range per image $\left(^{\circ}\right)$ & 0.1 \\
Total rotation range $\left(^{\circ}\right)$ & 116 \\
Exposure time per image (s) & 0.1 \\
Space group & $I 4_{1} 22$ \\
$a, b, c(\AA)$ & $143.090,143.090,76.430$ \\
$\alpha, \beta, \gamma\left({ }^{\circ}\right)$ & $90.000,90.000,90.000$ \\
Resolution range $(\AA)$ & $67.42-2.34(2.40-2.34)$ \\
No. of unique reflections & $17040(1246)$ \\
Completeness $(\%)$ & $99.95(100)$ \\
Multiplicity & $8.51(8.52)$ \\
$\langle I / \sigma(I)\rangle$ & $22.4(3.28)$ \\
$R_{\text {merge }}$ & $0.05(0.63)$ \\
$C_{1 / 2}$ & $99.9(75.4)$ \\
\hline
\end{tabular}

and Arg314; Feng et al., 2014). This AMP regulation differs from both GlpX, which is insensitive to AMP, and mammalian FBPases, in which AMP can bind directly to the active site (Xue et al., 1994). In a sequence alignment, all key residues are identical between T. elongatus and Synechocystis sp. PCC 6803 for the cysteine disulfide bridge and AMP-regulatory region.

This paper presents the structure of the type II FBP/SBPase from $T$. elongatus bound with sedoheptulose-7-phosphate and in the absence of AMP.

\section{Materials and methods}

\subsection{Macromolecule production}

The full-length FBPase/SBPase gene (tll1276) was cloned downstream of a $6 \times$ His tag in a modified pRSET A vector encoding a thrombin cleavage site. Recombinant FBP/SBPase was expressed in Escherichia coli KRX cells and purified on a nickel-affinity column; the His tag was then cleaved at the thrombin site (MRGSHHHHHHGLVPRG/S) and the protein was maintained in $50 \mathrm{~m} M$ Tris- $\mathrm{HCl}$ buffer $\mathrm{pH} 7.9$ with $50 \mathrm{~m} M$ $\mathrm{NaCl}$. No further purification steps were necessary for crystallization. Macromolecule-production information is summarized in Table 1.

\subsection{Crystallization}

Purified $\mathrm{FBP} / \mathrm{SBP}$ ase was stored at $4^{\circ} \mathrm{C}$ and a stock concentration of $15 \mathrm{mg} \mathrm{ml}^{-1}$ was used for crystallization trials. In the crystallization condition $0.1 M$ HEPES $\mathrm{pH} 7.5,0.8 M$ sodium dihydrogen phosphate, $0.8 M$ potassium dihydrogen phosphate, hexagonal bipyramidal crystals were found which were shown to belong to space group $P 6_{2} 22$ or $P 6_{4} 22$ but diffracted anisotropically to low resolution and were not processed further. Square bipyramidal crystals found in the condition in Table 2 [0.06 $M$ Na HEPES pH 7.5, $0.12 \mathrm{M} \mathrm{MgCl}_{2}$, $27 \%(v / v)$ PEG 400] diffracted to $2.3 \AA$ resolution and were used for the structure presented here. $20 \%(v / v)$ glycerol was added to the mother liquor as a cryoprotectant. Sedoheptu- 
Table 4

Structure refinement.

Values in parentheses are for the outer shell.

Resolution range $(\AA)$
Completeness $(\%)$
No. of reflections, working set
No. of reflections, test set
Final $R_{\text {cryst }}$
Final $R_{\text {free }}$
No. of atoms
Protein
Ion
Ligand
Water
Total
R.m.s. deviations
Bonds $(\AA)$
Angles $\left({ }^{\circ}\right)$
Ramachandran plot
Favoured $(\%)$
Additionally allowed $(\%)$
Outliers $(\%)$
MolProbity score

$67.4-2.34(2.49-2.34)$
$99.88(100)$
$16166(2645)$
$862(151)$
$0.166(0.206)$
$0.224(0.290)$
2537
8
18
74
2637

0.007
1.054
98
2
0
$1.30[100$ th percentile at
$\quad 2.34 \pm 0.25$ A resolution]

lose-7-phosphate (magnesium salt; $\mathrm{MgS7P}$ ) was added to the hanging drop at a concentration of $1 \mathrm{~m} M$ for co-crystallization studies.

\subsection{Data collection and processing}

Data were collected under cryocooled conditions on beamline I04 at the Diamond Light Source. All images from the synchotron were processed by xia2 (Winter, 2009) using the 3dii setting/XDS (Kabsch, 2010). Data-collection and processing statistics are presented in Table 3.

\subsection{Structure solution and refinement}

The structure was solved by molecular replacement using Phaser (McCoy et al., 2005) with E. coli GlpX in complex with fructose-1,6-phosphate (PDB entry 2r8t; Brown et al., 2009) as the model. Structures were refined using REFMAC (Murshudov et al., 2011) and tools from the CCP4 package (Winn et al., 2011) and were finished in PHENIX (Adams et al., 2010). Model building was performed in Coot (Emsley \& Cowtan, 2004). MolProbity was used for validation (Chen et al., 2010). Structure-solution and refinement statistics are presented in Table 4.

\section{Results and discussion}

The crystals belonged to space group $I 4_{1} 22$. The asymmetric unit contains a monomer, with a solvent content of $53 \%$. All loop regions are visible in the structure and are resolved, with the exception of ten residues at the C-terminus which are structured in the cyanobacterial dual-function FBP/SBPase structure with AMP bound. The structure appears to be an adventitiously purified product complex, as not only is the sedoheptulose-7-phosphate visible but an additional phosphate close to the $\mathrm{C}_{1}$ position of the sugar is also visible in the electron density at the active site. In contrast to previous cyanobacterial structures, no adenine mononucleotide phosphate (AMP) is bound, and the C-terminal tail that binds AMP is not visible in the electron density.

Superposition of $\mathrm{C}^{\alpha}$ atoms of the structure of the Synechocystis sp. PCC 6803 FBP/SBPase bound with fructose-1,6bisphosphate and AMP (PDB entry 3rpl) and the T. elongatus structure bound with sedoheptulose-7-phosphate (PDB entry 5a51) shows a root-mean-square deviation (r.m.s.d.) of $0.727 \AA$. The two structures are very similar. This value changes to $0.740 \AA$ for the comparison with the Synechocystis structure without the sugar substrate bound (PDB entry 3roj), implying that the binding of the substrate does not result in any global conformational change. Both proteins show the two $\alpha / \beta$-sandwich domains of the $\mathrm{Li}^{+}$-sensitive phosphatases.

All active-site residue identities (Asp33, Glu57, Glu100, Thr102, Tyr131, Lys134, Arg176, Arg178, Asp198, Asp200 and Glu225) and positions are conserved between the Synechocystis sp. PCC 6803 structure and the T. elongatus structure bound with different sugars. The metals (magnesium ions) are

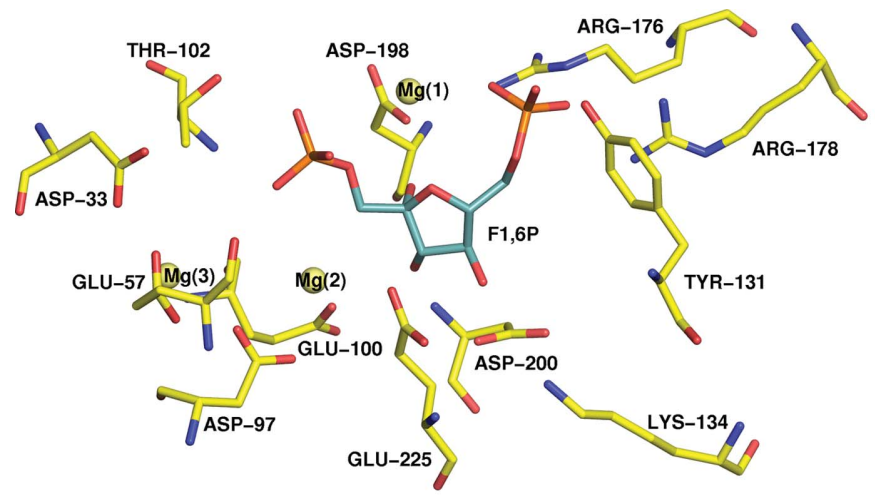

(a)

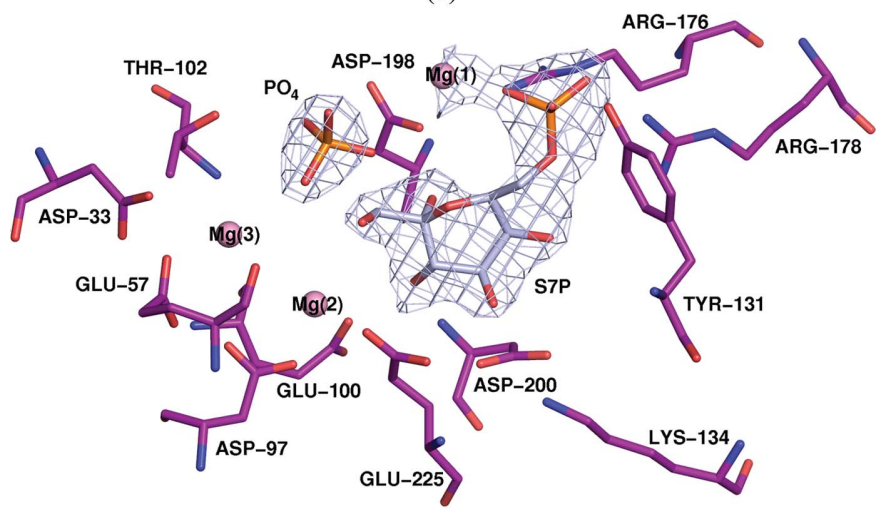

(b)

Figure 1

Comparison of similar active sites. (a) Synechocystis sp. PCC 6803 structure (PDB entry 3rpl, yellow) shown with key active-site residues shown as sticks (Asp33, Glu57, Glu100, Thr102, Tyr131, Lys134, Arg176, Arg178, Asp198, Asp200 and Glu225). (b) T. elongatus structure (PDB entry 5a5l, purple) bound with sedoheptulose-7-phosphate (lavender) with equivalent active-site residues shown as sticks (Asp33, Glu57, Glu100, Thr102, Tyr131, Lys134, Arg176, Arg178, Asp198, Asp200 and Glu225). Magnesium ions are shown as spheres. The phosphate $\left(\mathrm{PO}_{4}\right)$ of the suggested product complex is clearly visible. Ligand OMIT map difference density is shown for the S7P ligand and phosphate (lavender mesh) using a $4 \sigma$ contour level. Active-site residues and metals are in the same conformation; selectivity between the sugars is not the result of large-scale conformational change. 
shifted by $1.9,2.0$ and $3.4 \AA$, respectively, for magnesiums 1,2 and 3 (Fig. 1) in the two structures but are bound to the same residues. The phosphate in the T. elongatus structure suggests that the product complex has been purified as it resides roughly at the equivalent ' 1 ' position of the fructose-1,6bisphosphate and is located next to the $\mathrm{C} 1$ atom of sedoheptulose-7-phosphate. There does not appear to be a structural mechanism to differentiate between sugars.

Redox regulation is thought to be important for type II FBPases involved in photosynthesis (Feng et al., 2014). The mechanism of redox regulation in cyanobacterial FBP/ SBPases is clearly distinct from that in FBPase structures from the higher plant Pisum sativum (PDB entries 1d9q and 1dbz; Chiadmi et al., 1999), in which the redox-active disulfide is on a loop covering the active site. Feng and coworkers suggest that cysteines at positions Cys 75 and Cys99 may rearrange to form a disulfide bond in the inactive enzyme state using evidence from a targeted mutagenesis activity assay (Feng et al., 2014). In both the T. elongatus structure and the Synechocystis sp. PCC 6803 structure the only oxidized disulfide bond appears to be Cys75-Cy84. Our structure does not provide evidence for the hypothesis of Feng and coworkers, as the two residues are too far apart in this conformation and would require significant conformational change to the 'inactive' state. In an

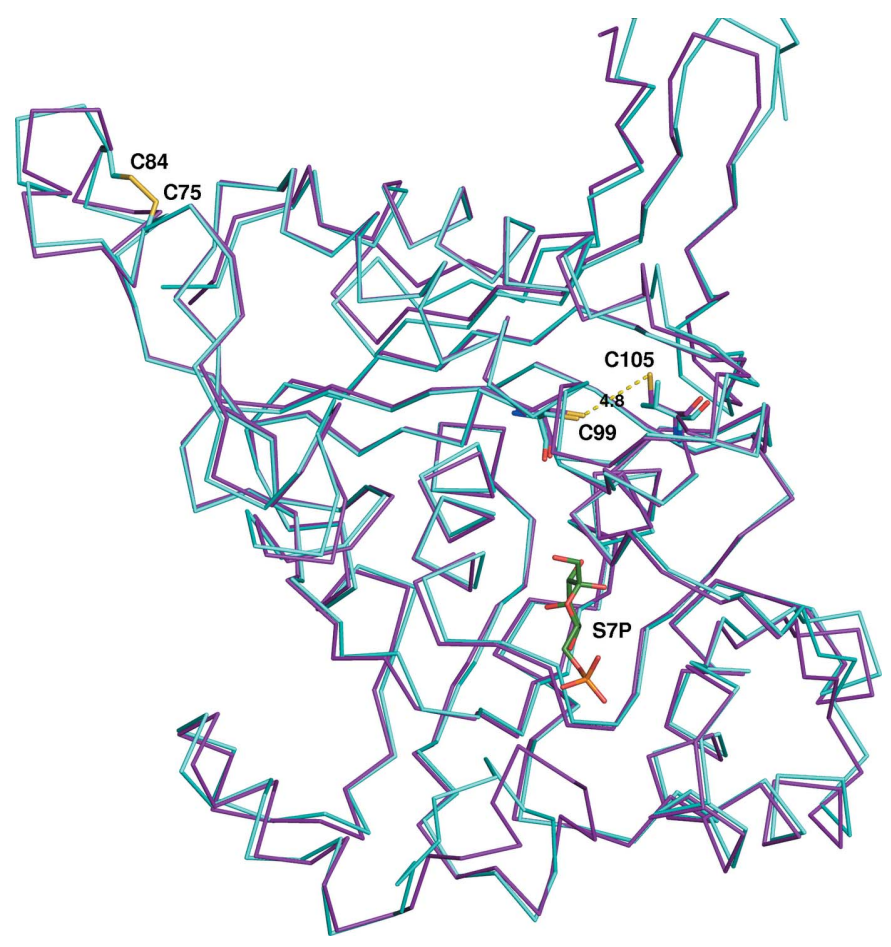

Figure 2

Redox regulation. The residues previously identified as being important for redox regulation in Synechocystis sp. PCC 6803 (cyan) are Cys75 and Cys99, which are too far away to be involved in a disulfide bond in the current conformation. Cys 75 and Cys 84 are oxidized in both structures. In addition, Cys 105 could potentially form a disulfide bond with Cys 99 in the T. elongatus protein (dashed line, $4.8 \AA$ ). This Cys105 is not conserved and is a valine in the Synechocystis protein. Sedoheptulose-7-phosphate (S7P, green) is shown in the image to highlight the distance of these residues from the active site. The $T$. elongatus structure is shown as a ribbon (purple). alignment of the T. elongatus FBP/SBPase and the Synechocystis sp. PCC 6803 FBP/SBPase all cysteines were conserved in both position and structural orientation, with four exceptions: Cys235, Cys281 and Cys288 were not present in T. elongatus and Cys105 was not present in Synechocystis sp.

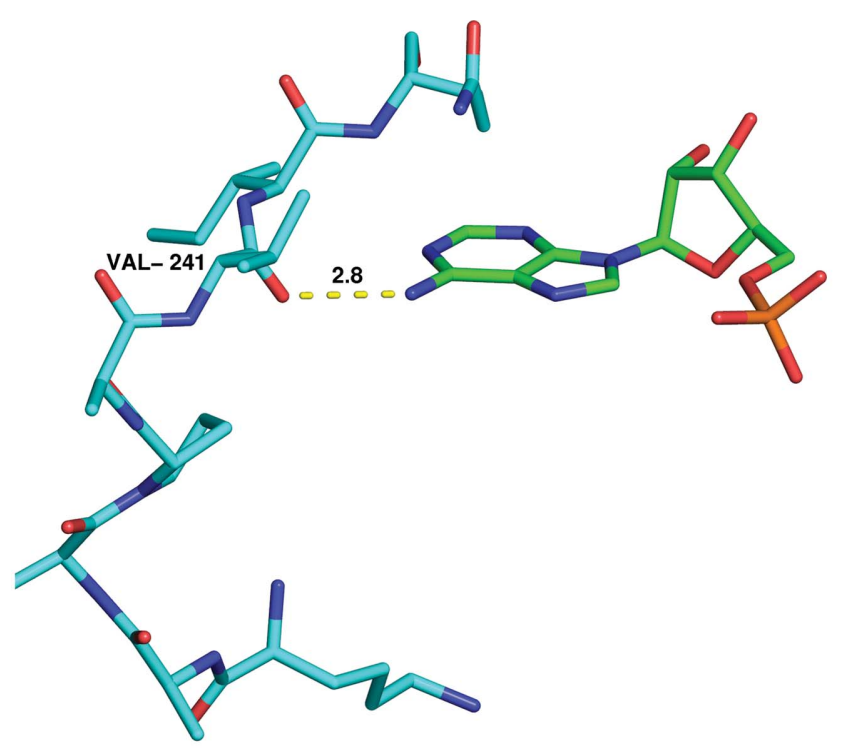

(a)

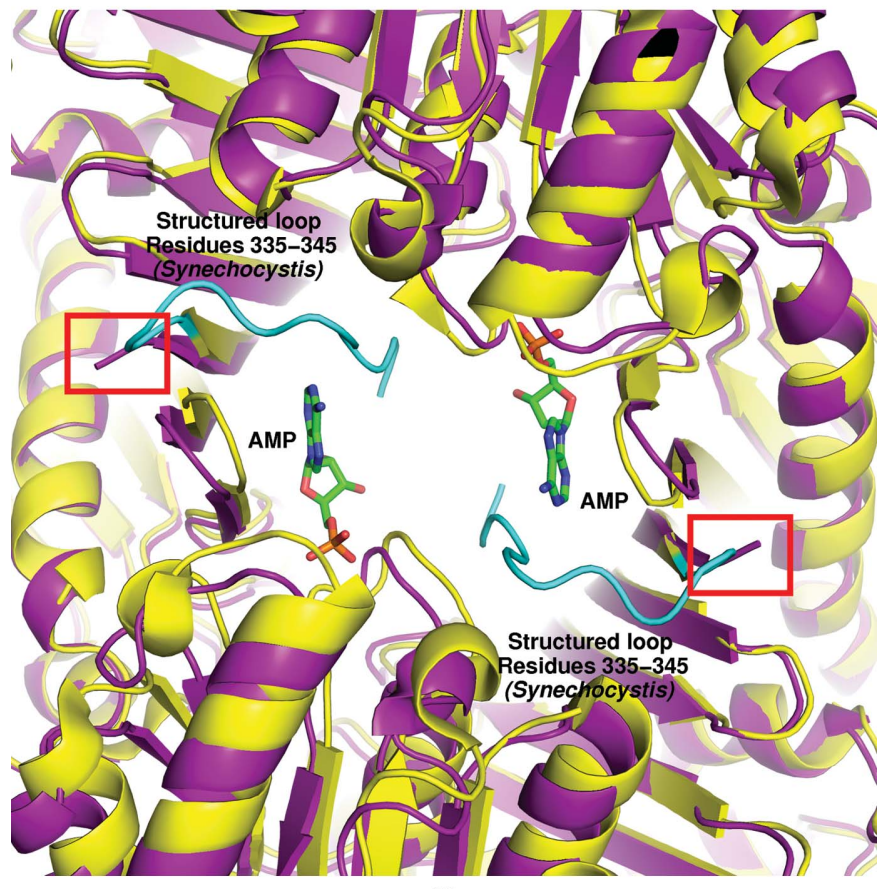

(b)

Figure 3

The AMP-binding loop found in the Synechocystis sp. PCC 6803 structure is not visible in the T. elongatus electron density in the absence of AMP. (a) Stick model of Synechocystis sp. PCC 6803 residues 335-345 (cyan), which form a structured loop in the presence of AMP (green). The backbone carboxyl of Val341 forms a hydrogen bond to AMP. (b) AMP (green) is bound only in the Synechocystis sp. PCC 6803 structure (yellow cartoon). The final ten residues (structured loop) of the Synechocystis sp. PCC 6803 structure are shown in cyan as in $(a)$ to indicate that they form part of the AMP-binding region. The purple cartoon is the T. elongatus FBP/SBPase. The red box indicates the end of the T. elongatus structure, as the final residues are not visible in the electron density. 
PCC 6803. The cysteines at positions Cys75, Cys84 and Cys99 are well conserved in a survey of cyanobacterial FBP/SBPase sequences. In the T. elongatus sequence Cys 105 is positioned sufficiently close to Cys99 to form a disulfide bond (see Fig. 2) but is not oxidized in this structure, and this cysteine is not generally conserved in cyanobacteria. In conclusion, no structural evidence for the rearrangement hypothesis of Feng and coworkers can be seen in our structure lacking AMP (Feng et al., 2014).

The AMP-binding domain has key residues Arg164, Asn213, His215, Arg307, Phe309 and Arg314 in the Synechocystis sp. PCC 6803 structure (Feng et al., 2014). All of the key residues identified are conserved in our sequence and are almost identical in both position and orientation in the structure. One critical difference appears to be the ordering of the final residues of the $\mathrm{C}$-terminus. Phe 337 is the final residue that is visible in the electron density of the T. elongatus structure. Feng and coworkers reported that the Synechocystis sp. PCC 6803 enzyme would not crystallize in the absence of AMP. This is presumably because this region (see Fig. 3, cyan) was unstructured and disrupted crystallization. While the full sequence of the loops is not well conserved, there is a clear hydrogen-bonding interaction between the backbone carboxyl of Val341 and the nitrogenous base (the amino group extending from the $\mathrm{C} 6$ atom of the adenine moiety), which is not resolved in our structure.

The main contribution of this structure, which is globally very similar to the Synechocystis sp. PCC 6803 enzyme, is the evidence for disorder of the AMP-binding region in the absence of AMP. In addition, the structure provides further information for understanding selectivity at the active site and supports the assertion that the mechanism of redox regulation is not yet structurally evident.

\section{Acknowledgements}

The authors would like to thank Diamond Light Source for beamtime (proposal mx7299) and the staff of beamline I04 for assistance with crystal testing and data collection. CC is supported by a BBSRC Scholarship. JWM was supported by a David Phillips Fellowship for part of this work (BB/F023308/1).

\section{References}

Adams, P. D. et al. (2010). Acta Cryst. D66, 213-221.

Brown, G., Singer, A., Lunin, V. V., Proudfoot, M., Skarina, T., Flick, R., Kochinyan, S., Sanishvili, R., Joachimiak, A., Edwards, A. M., Savchenko, A. \& Yakunin, A. F. (2009). J. Biol. Chem. 284, 37843792.

Chen, V. B., Arendall, W. B., Headd, J. J., Keedy, D. A., Immormino, R. M., Kapral, G. J., Murray, L. W., Richardson, J. S. \& Richardson, D. C. (2010). Acta Cryst. D66, 12-21.

Chiadmi, M., Navaza, A., Miginiac-Maslow, M., Jacquot, J.-P. \& Cherfils, J. (1999). EMBO J. 18, 6809-6815.

Emsley, P. \& Cowtan, K. (2004). Acta Cryst. D60, 2126-2132.

Feng, L., Sun, Y., Deng, H., Li, D., Wan, J., Wang, X., Wang, W., Liao, X., Ren, Y. \& Hu, X. (2014). FEBS J. 281, 916-926.

Kabsch, W. (2010). Acta Cryst. D66, 125-132.

McCoy, A. J., Grosse-Kunstleve, R. W., Storoni, L. C. \& Read, R. J. (2005). Acta Cryst. D61, 458-464.

Miyagawa, Y., Tamoi, M. \& Shigeoka, S. (2001). Nature Biotechnol. 19, 965-969.

Murray, J. W., Maghlaoui, K. \& Barber, J. (2007). Acta Cryst. F63, 998-1002.

Murshudov, G. N., Skubák, P., Lebedev, A. A., Pannu, N. S., Steiner, R. A., Nicholls, R. A., Winn, M. D., Long, F. \& Vagin, A. A. (2011). Acta Cryst. D67, 355-367.

Poolman, M. G., Ölçer, H., Lloyd, J. C., Raines, C. A. \& Fell, D. A. (2001). Eur. J. Biochem. 268, 2810-2816.

Sun, Y., Zhang, R., Li, D., Feng, L., Wu, D., Feng, L., Huang, P., Ren, Y., Feng, J., Xiao, S. \& Wan, J. (2014). J. Chem. Inf. Model. 54, 894-901.

Tamoi, M., Ishikawa, T., Takeda, T. \& Shigeoka, S. (1996). Arch. Biochem. Biophys. 334, 27-36.

Tamoi, M., Murakami, A., Takeda, T. \& Shigeoka, S. (1998). Biochim. Biophys. Acta, 1383, 232-244.

Tamoi, M., Takeda, T. \& Shigeoka, S. (1999). Plant. Cell. Physiol. 40, 257-261.

Winn, M. D. et al. (2011). Acta Cryst. D67, 235-242.

Winter, G. (2009). J. Appl. Cryst. 43(1), 186-190.

Xue, Y., Huang, S., Liang, J.-Y., Zhang, Y. \& Lipscomb, W. N. (1994). Proc. Natl Acad. Sci. USA, 91, 12482-12486. 